\title{
Identification of Transient Receptor Potential Channel 4-Associated Protein as a Novel Candidate Gene Causing Congenital Primary Hypothyroidism
}

\author{
Daniela Choukair ${ }^{a} \quad$ Birgit Eberle $^{b}$ Philipp Vick ${ }^{c}$ Pia Hermanns ${ }^{d}$ Birgit Weiss ${ }^{b}$ \\ Nagarajan Paramasivam ${ }^{\mathrm{e}}$ Matthias Schlesner ${ }^{f}$ Katharina Lornsen $^{\mathrm{g}}$ \\ Ralph Roeth $^{\mathrm{b}}$ Carina Klutmann ${ }^{\mathrm{d}}$ Jennifer Kreis ${ }^{\mathrm{c}}$ Georg F. Hoffmann ${ }^{\mathrm{a}}$ \\ Joachim Pohlenz ${ }^{d}$ Gudrun A. Rappold ${ }^{b}$ Markus Bettendorfa

\begin{abstract}
a Division of Paediatric Endocrinology, Children's Hospital, University of Heidelberg, Heidelberg, Germany; ${ }^{b}$ Department of Human Molecular Genetics, University of Heidelberg, Heidelberg, Germany; ${ }^{C}$ Department of

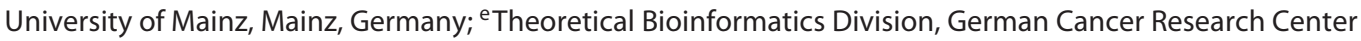
(DKFZ), Heidelberg, Germany; ${ }^{f}$ Bioinformatics and Omics Data Analytics (B240), German Cancer Research Center (DKFZ), Heidelberg, Germany; ${ }^{9}$ Genomics and Proteomics Core Facility, German Cancer Research Center (DKFZ), Heidelberg, Germany
\end{abstract} \\ Zoology, University of Hohenheim, Stuttgart, Germany; d Division of Paediatric Endocrinology, Children's Hospital,
}

\begin{abstract}
Background: Congenital primary hypothyroidism $(\mathrm{CH})$ is the most common endocrine disorder in neonates. Methods: To identify novel genes, we performed whole exome sequencing (WES) in 6 patients with $\mathrm{CH}$ due to thyroid dysgenesis (TD). The potential effects of the most relevant variants were analyzed using in silico prediction tools. The most promising candidate gene, transient receptor potential channel 4-associated protein (TRPC4AP), was sequenced in 179 further patients with TD. Expression of TRPC4AP in human thyroid was investigated using RT-PCR. Trpc4ap- functional analysis was performed in Xenopus laevis using Morpholino (MO) antisense oligomers. Results: WES identified a likely damaging mutation in TRPC4AP leading to a de novo stop codon $\mathrm{p}$.
\end{abstract}

Q552*. Targeted sequencing of TRPC4AP demonstrated gene variants with predicted damaging potential in 5 patients resulting each in an amino acid exchange (p.P706S, p.F729L, p.S777C, and p.N229S). We demonstrated that TRPC4AP is expressed in human thyroid gland tissue. Using Xenopus laevis, we showed that the volume of the tadpole thyroid anlage was reduced by $20 \%$ in Trpc4ap MO knockdowns compared to controls and by $41 \%$ in "Clustered Regularly Interspaced Short Palindromic Repeats"/Cas9-mediated gene knockout experiments. Discussion: A recognized interaction of TRPC4AP and the NF-kappa-B-essential-modulator encoded by IKBKG gene was identified by IPA analysis. IKBKG plays a role in activation of the NF-KB-signaling pathway and regulates genes involved in proliferation and sur-

D.C and B.E. as well as G.A.R. and M.B. contributed equally to this work and should therefore be both considered as first and senior coauthor, respectively. 
vival of thyrocytes and expression of key enzymes of thyroid hormone synthesis. Conclusion: TRPC4AP was identified as a novel candidate gene in TD, but further studies are needed to validate its role in thyroid function. $\odot 2020$ S. Karger AG, Basel

\section{Introduction}

Congenital primary hypothyroidism $(\mathrm{CH})$ is the most common endocrine disorder in neonates, affecting one in 2,500-3,500 newborns [1]. After introduction of the newborn screening in Germany in the 1970s, the symptoms of $\mathrm{CH}$ such as growth retardation and delay of psychomotor development can be prevented by an early treatment start with L-thyroxin [2]. In the vast majority of patients, $\mathrm{CH}$ is caused by developmental thyroid abnormalities, known as thyroid dysgenesis (TD), comprising athyreosis, thyroid ectopia, thyroid hypoplasia, and hemithyroidosis [1]. During embryogenesis, the midline anlage appears on embryonic day E22 developing from the floor of the foregut [3]. The ultimobranchial bodies develop from the fourth pharyngeal pouch on each side from E26 onward. The median and lateral anlagen migrate and later fuse further at the definitive pretracheal position at E44 [3]. Cells differentiate into thyrocytes expressing thyreoglobulin (Tg) and producing thyroxine (T4) or into Ccells expressing calcitonin at gestational week 8. Abnormalities in thyroid development at any step such as differentiation, cell migration, fusion, and survival can lead to TD. So far, 10 genes with various clinical spectra have been identified in sporadic and familial TD: $P A X 8$, NKX21, FOXE1, probably NKX2-5, TSHR, GLIS3, NTN1, JAG1, CDCA8, and TUBB1 [4-6]. However, these mutations are detected in only $5 \%$ of patients with $\mathrm{CH}$. Identification of damaging mutations remains a challenging task. In this study, we aimed to identify novel gene candidates causing $\mathrm{CH}$ by whole exome sequencing (WES) in 6 unrelated patients with TD and their respective parents.

\section{Materials and Methods}

\section{Patients and Controls}

All patients who were included in this study were identified in the newborn screening program at the University Children's Hospital Heidelberg by elevated thyroid-stimulating hormone (TSH) concentrations in whole blood dry spots. The diagnoses of $\mathrm{CH}$ were confirmed in the Division of Paediatric Endocrinology and Diabetes of the University Children's Hospital in Heidelberg $(n=$ 153; 86 females), in the Division of Paediatric Endocrinology and
Diabetes of the University Children's Hospital in Mainz $(n=23$; 13 females), or decentralized ( $n=9$; 5 females) by local pediatricians who then initiated adequate treatment with L-thyroxine after informed consent was given by the parents [7] and transferred peripheral blood for genetic analyses. Therefore, phenotypes are not transparent in all details and may have been reported incompletely or could not be verified (patients HD-98; HD-131) when patients were not followed in our children's Hospital. Six patients followed in Heidelberg ( 3 females and 3 males), and their parents were randomly recruited from a cohort of consecutive patients with $\mathrm{CH}$ followed in our institution with their informed consent for WES.

Syndromic or extrathyroidal malformations were absent in this subgroup. None of the patients had defective gland migration. For clinical charcaterization see Table 1 and online supplementary Table S1 (see www.karger.com/doi/10.1159/000507114 for all online suppl. material). Location of thyroid gland was pictured by ultrasound including color Doppler. DNA was extracted from peripheral blood leucocytes using the salting out method. Finally WES was performed in 18 samples of patients and their respective parents (6 trios).

DNA samples of additional 156 (91 females) patients treated in our institution and 23 (13 females) patients treated in the Division of Paediatric Endocrinology and Diabetes of the University Children's Hospital in Mainz with TD had been collected with informed consent of their parents for targeted sequencing. Sequencing data from the gnomAD Server (https://gnomad.broadinstitute. org) and 1000 Genomes (http://www.1000genomes.org/) were used as controls.

\section{WES and Filtering}

The Agilent SureSelect Human All Exon V4 (without UTR) was utilized to capture the exons according to the manufacturer's recommendations. Sequencing was done using the Illumina HiSeq 2000 system. The raw sequence data were mapped to the 1,000 genomes phase II reference genome (hs37d5) using BWA 0.6.2, and the duplicates were removed using PICARD. The 18 samples showed average exon coverage of $95.872 \times$ and a medianof-median exon coverage of $90.32 \times$. About $99.904 \%$ of exons showed nonzero coverage, and $99.252 \%$ of exons had at least $10 \times$ coverage. On average, the bases in the target regions with QUAL $>1$ showed a coverage of $105.575 \times$ with $99.026 \%$ showing a minimum of $10 \times$ coverage and $97.068 \%$ a minimum of $20 \times$ coverage. The single nucleotide variants (SNVs) were called by SAMtools on individual samples, and the respective parent information was merged later. Indels were then called along with parent samples using Platypus [8]. In-house pipelines were utilized to annotate the variants with dbSNPs and 1,000 genomes database. ANNOVAR [9] and RefSeq were used for the functional annotation of the variants.

SNVs and indels with a read depth of at least $10 \times$ and minimum QUAL score of 20 were considered for further analysis. Nonsynonymous (missense, stop gain, stop loss) and splice site affecting SNVs as well as all exonic indels were filtered further. Custom variant allele frequency (VAF) thresholds were used to assign genotypes to the SNVs. For SNVs to be heterozygous, they should have a minimum of $15 \% \mathrm{VAF}$ and a maximum of $90 \% \mathrm{VAF}$. SNVs with VAF of $95 \%$ and above were considered as homozygous alternate and those with VAF of 5\% and below as homozygous reference. For Indels, the genotype was predicted by Platypus. 
Table 1. Patient characteristics for the cohort selected for WES

\begin{tabular}{|c|c|c|c|c|c|c|c|c|c|c|c|c|c|}
\hline Patient & $\begin{array}{l}\mathrm{CA} \text {, } \\
\text { years }\end{array}$ & Gender & $\begin{array}{l}\text { Screening } \\
\text { TSH, IU/L }\end{array}$ & $\begin{array}{l}\text { Serum } \\
\text { TSH, } \\
\text { IU/L }\end{array}$ & $\begin{array}{l}\text { Serum } \\
\text { T3, } \\
\text { nmol/L }\end{array}$ & $\begin{array}{l}\text { Serum } \\
\mathrm{T} 4 \text {, } \\
\mathrm{nmol} / \mathrm{L}\end{array}$ & $\begin{array}{l}\text { Serum } \\
\text { fT4, } \\
\text { pmol/L }\end{array}$ & $\begin{array}{l}\text { Serum } \\
\text { thyroglobulin, } \\
\mathrm{ng} / \mathrm{mL}\end{array}$ & Ultrasound & $\begin{array}{l}\text { Follow- } \\
\text { up at } \\
\text { CA }\end{array}$ & $\begin{array}{l}\text { Serum } \\
\text { thyroglobulin, } \\
\mathrm{ng} / \mathrm{mL}\end{array}$ & Ultrasound & $\begin{array}{l}\text { Actual } \\
\text { L-Thyroxine } \\
\text { dosage, } \mu \mathrm{g} / \\
\mathrm{kg} / \text { day }\end{array}$ \\
\hline 1 & 9 & Female & 236 & $>100$ & uk & uk & uk & 21 & Hypoplasia & 2 & 2.9 & Athyreosis & 2.7 \\
\hline 2 & 8 & Female & 603 & 603.6 & $\mathrm{uk}$ & $\mathrm{uk}$ & 8.21 & 9.2 & Athyreosis & 4 & 8.2 & Athyreosis & 2.8 \\
\hline 3 & 15 & Female & 342 & $>150$ & 1.4 & 75.3 & 9.6 & 42 & Hypoplasia & 14 & 0.82 & Athyreosis & 2 \\
\hline 4 & 13 & Male & 256 & $>150$ & 0.4 & 5 & $\mathrm{uk}$ & $<0.15$ & Athyreosis & 5 & 0.2 & Athyreosis & 2.1 \\
\hline 5 & 4 & Male & 120 & nd & $\mathrm{uk}$ & uk & $\mathrm{uk}$ & uk & Hypoplasia & 4 & 1.8 & Athyreosis & 4.1 \\
\hline 6 & 12 & Male & 255 & $>150$ & 1.2 & 31.4 & 5.6 & 41 & $\begin{array}{l}\text { Normal } \\
\text { thyroidea }\end{array}$ & 2 & 2.8 & Athyreosis & 2 \\
\hline
\end{tabular}

Actual patient CA is given. Postnatal concentrations of screening TSH, of serum TSH, T3, T4, fT4, thyroglobulin, and morphology examined by ultrasound are depicted. Further, chronological age of follow-up is indicated, when a confirming ultrasound was performed and serum thyroglobulin was measured while serum TSH concentrations were elevated. Further, the actual L-thyroxine dosage is indicated. Reference thyroid volume in neonates $1.1 \pm 0.8$ $\mathrm{mL}$. CA, chronological age; TSH, thyroid-stimulating hormone; WES, whole exome sequencing; uk, unknown.

The minor allele frequency (MAF) from 1,000 genomes database was used to define the variant's rareness in the population. Variants with MAF $>1 \%$ were removed (removal of compound heterozygous variants if MAF of one allele was $>1 \%$ ). Furthermore, a set of 45 samples of WES from the in-house database was used as control to remove common variants and sequencing artifacts (removal of compound heterozygous variants if one or both alleles were found in the in-house controls).

The functional effect of the remaining variants was predicted using the in silico prediction tools MutationTaster, SIFT, PolyPhen2, Provean, and Condel. In addition, the gnomAD browser, CADD score, and intolerance scores (from gnomAD) were included to characterize the identified variants. Variants that were predicted as damaging by at least one in silico prediction tool were used for further analysis. Compound heterozygous mutations were only included if both variants were predicted as damaging by at least one prediction tool.

\section{RNA Isolation of the Thyroid Gland}

Total RNA from a human thyroid gland after strumectomy due to Graves' disease was extracted using the FastGene RNA Premium kit (Nippon Genetics, Düren, Germany). Two micrograms of the total RNA was reverse transcribed using the reverse transcription systems from Promega (Mannheim, Germany) following the manufacturer's recommendations. The cDNA was amplified with the primers for transient receptor potential channel 4-associated protein (TRPC4AP; forward, $5^{\prime}$-GGT CCG GGC GGT GCA GTT CAC TGA GAC-03; reverse, 5'-GAG ATC GTA GGC CTC ATA AGA ATT TC- $3^{\prime}$ ) and for thyroid peroxidase (TPO; forward, $5^{\prime}$-CAG AAG AGT TAC AGC CGT GA-3'; reverse, $5^{\prime}$-ATT ATC TCT GCT GCT CGG GC-3'), respectively. For PCR amplification the Axon Taq Polymerase system was utilized (Axon Labortechnik, Kaiserslautern, Germany). The PCR products were electrophoretically separated on a $2 \%$ agarose gel and stained with MidoriGreen (Nippon Genetics, Düren, Germany) and blue LED transilumination. The PCR products were also purified enzymatically with Exonuclease I (New England Biolabs, Frankfurt, Germany) and shrimp alka- line phosphatase (GE Healthcare, München, Germany), then sequenced with BigDye terminator version 3.1 kit followed by ethanol precipitation, and then analyzed using an automated sequencing system (3130 Avant Genetic Analyzer, Applied Biosystems, Darmstadt, Germany) to show that the amplified PCR products are gene specific.

\section{Xenopus Laevis}

Xenopus Laevis Care and Maintenance

Frogs were purchased from Nasco (901 Janesville Avenue PO Box 901 Fort Atkinson). Handling, care, and experimental manipulations of animals were approved by the Regional Government Stuttgart, Germany (Vorhaben A379/12 ZO "Molekulare Embryologie"), according to German regulations and laws ( $\$ 6$, article 1 , sentence 2 , number 4 of the animal protection act). Animals were kept at the appropriate conditions $\left(\mathrm{pH}=7.7,20^{\circ} \mathrm{C}\right)$ at a $12-\mathrm{h}$ light cycle in the animal facility of the Institute of Zoology of the University of Hohenheim. Female frogs (4-15 years old) were injected subcutaneously with 300-700 units of human chorionic gonadotropin Sigma, depending on weight and age, to induce ovulation. Only clutches of embryos from healthy females were used for the experiments reported here - provided the early embryonic stages showed normal survival rates as well. Individual embryos from one batch were randomly picked and used either as control or tested specimens.

\section{In Situ Hybridization}

For trpc4ap in situ mRNA detection, whole-mount in situ hybridization was performed using a cloned full-length RNA probe. Embryos were fixed in MEMFA for 2-3 h at room temperature and processed following Xenopus in situ hybridization standard protocols [10]. RNA in situ probes were transcribed using SP6 or T7 polymerases.

\section{Morpholino Design and Microinjections}

A trpc4ab 5'UTR-Morpholino (MO) was designed using the sequence of the L-form (no S-form present) from the genomic sequence as deposited in gene bank (accession: NM_001096374). 


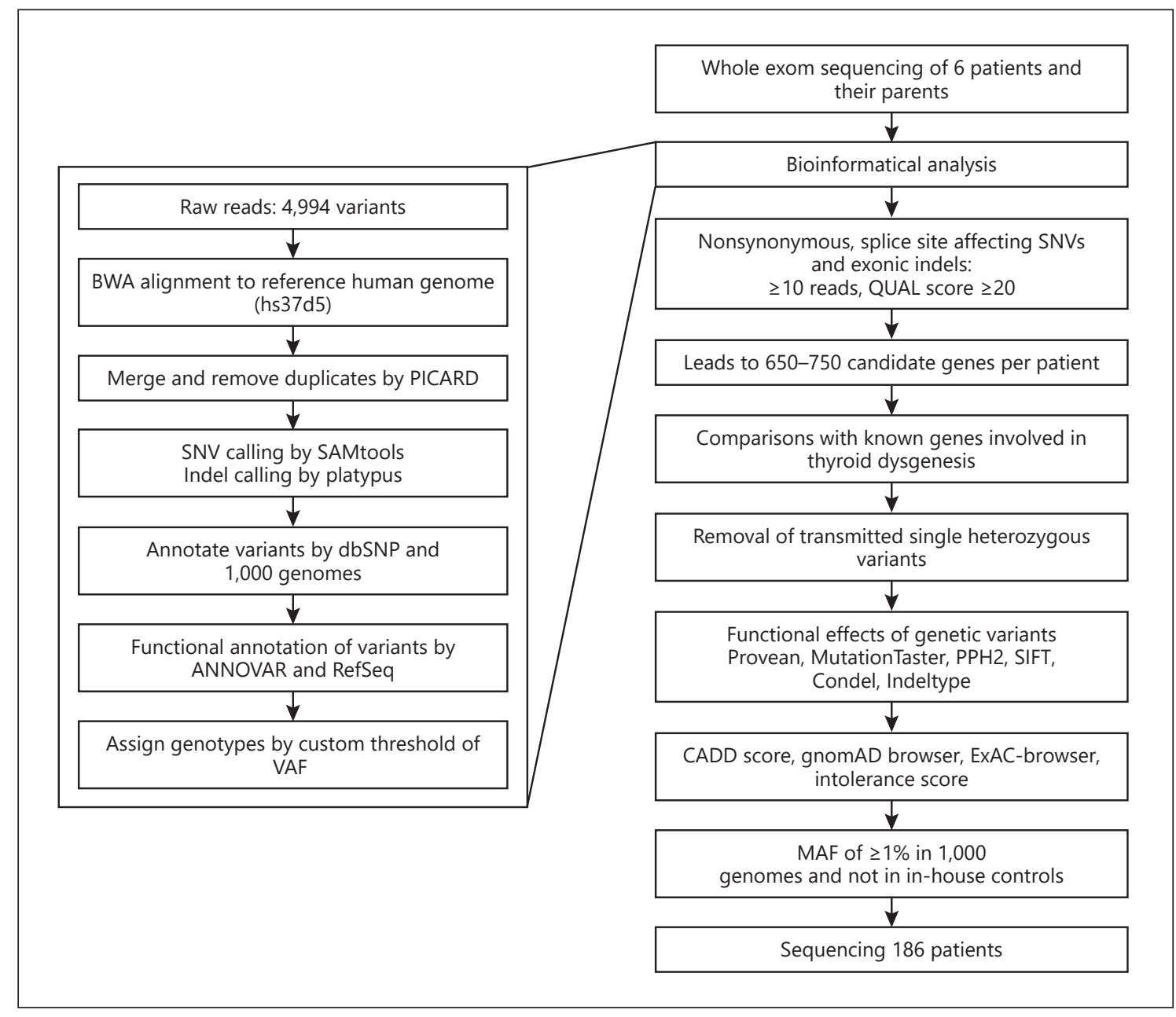

Fig. 1. Strategy to identify candidate genes involved in thyroidal gland development. Pipeline of WES and filtering. VAF, variant allele frequency; MAF, minor allele frequency.

The MO-sequence is $5^{\prime}$-GTACCGACCGCTATCAAGCAAGGAA-3'. Embryos were injected at the 4-cell stage into the dorsal blastomeres, either bilaterally or unilaterally left or right, targeting the future ventral foregut (i.e., including thyroid anlagen) using a Harvard Apparatus setup. Drop size was calibrated to $5 \mathrm{~nL} /$ injection. Amounts of injected MOs are indicated. Either uninjected littermates or uninjected halves of the same embryo served as (internal) controls.

\section{sgRNA Design and Microinjections}

A single-guide RNA (sgRNA; target sequence: 5'-GGGGGCAGCTGACGGGGTG-3') for exon 1 of the Xenopus trpc4ap gene (L-form) was designed using the publicly available "CRISPscan" software (11). sgRNAs were transcribed with the MEGAshortscript T7 Kit from synthetic DNA oligomers and purified with the MEGAclear Transcription Clean-Up Kit (both ThermoFisher). Oligos for synthesis were F-5'-GCAGCTAATACGA CTCACTATAGGGGGCAGCTGACGGGGTGGTTTTAGAGCTAGAAATAGCAAG-3' ${ }^{\prime}$ and R-5'-AAAAGCACCGACTCGGTGCCACTTTTTCAAGTTGATAACG-GACTAGCCT-
TATTTTAACTTGCTATTTCTAGCTCTAAAAC-3'. Embryos were injected with $1 \mathrm{ng}$ Cas9 protein (PNA Bio) and $300 \mathrm{pg}$ sgRNA at 1-cell stage, embryos were then cultivated at room temperature until stage 45 . DNA was isolated from 10 mutant or 5 control embryos. Uninjected littermates served as controls. EnGen Mutation Detection Kit (E3321; New England Biolabs) was used for T7 Endonuclease I based detection of Clustered Regularly Interspaced Short Palindromic Repeats/Cas9-induced mutations in targeted genomic sequences of the trpc4ap gene. PCR-based amplification reaction for DNA samples was after the manufacturer's protocol adapted for taq polymerase conditions. PCR oligos were as follows: F1-5'-CATCCCAGCATGCCCTTGTA- ${ }^{\prime}$ and R1-5'-GCAAGTCCATATAAATAAGGAAGCC- $3^{\prime}$. For heteroduplex formation, $1 \mu \mathrm{g}$ of amplified DNA was used. Initial denaturation was at $95^{\circ} \mathrm{C}$ for $5 \mathrm{~min}$, annealing at $95-85^{\circ} \mathrm{C}$ for $-2{ }^{\circ} \mathrm{C} / \mathrm{s}$, and $85-25^{\circ} \mathrm{C}$ for $-0.1^{\circ} \mathrm{C} / \mathrm{s}$. For heteroduplex digestion, $1 \mu \mathrm{L}$ of $\mathrm{T} 7$ Endonuclease was added at $37^{\circ} \mathrm{C}$ for $15 \mathrm{~min}$, followed by inactivation treated with $1 \mu \mathrm{L}$ Proteinase $\mathrm{K}$ for $5 \mathrm{~min}$ at $37^{\circ} \mathrm{C}$. Knockout efficiency was visualized by gel electrophoresis [11]. 


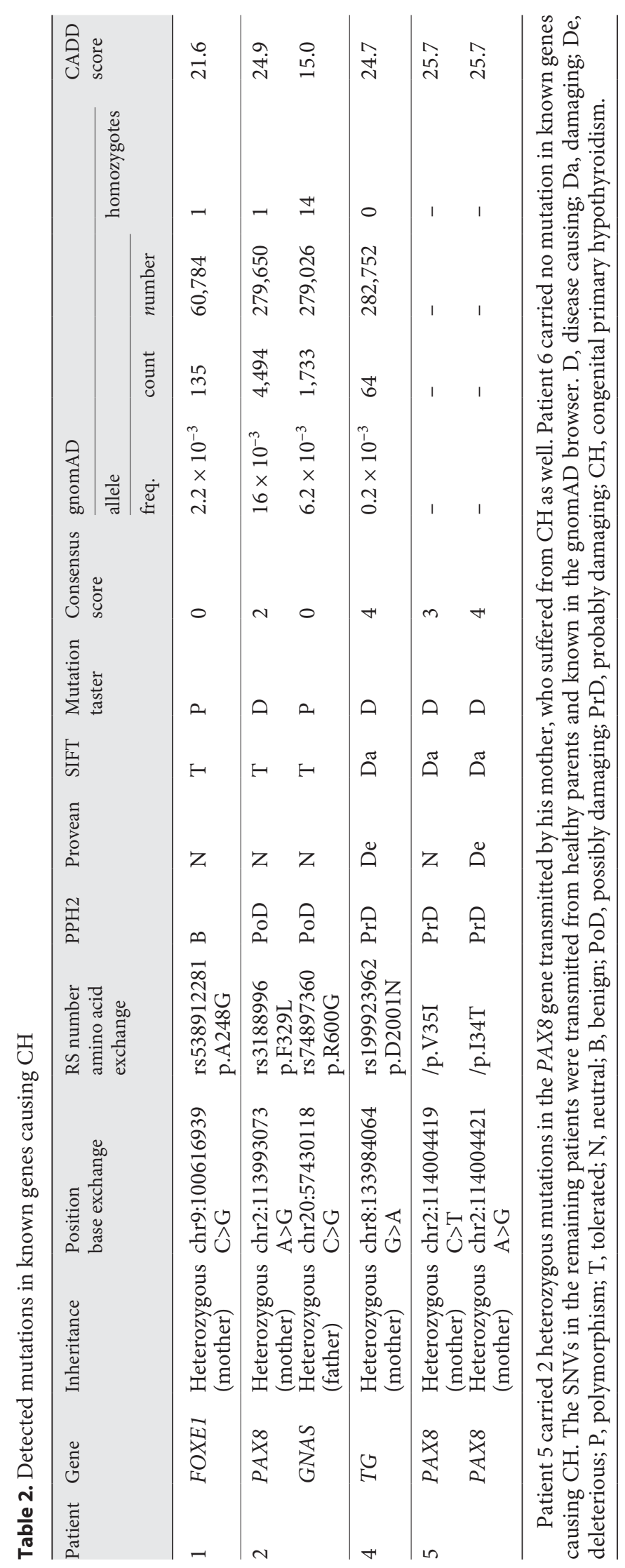

\section{Embryo Sections}

Embryos were fixed either in 4\% formaldehyde for $3 \mathrm{~h}$ or in Bouin solution over night or longer at room temperature. Sections for thyroid analyses were either made using a vibratome (Leica VT1000S; sections $40 \mu \mathrm{m}$ ) or a microtome (Reichert, Austria; sections $10 \mu \mathrm{m})$. For vibratome, embryos were embedded in a glutaraldehyde-crosslinked gelatin-albumin mix and razor blade-sectioned frontally. For microtome sections, embryos were dehydrated in isopropyl alcohol and embedded in paraffin wax blocks. Paraffin sections were then stained with "nuclear fast red" (Roth, Karlsruhe, Germany).

\section{Analyses of Thyroid Anlagen}

Quantification of thyroid sizes was performed at stage 45, when early split anlagen are found bilaterally embedded in the ventral suprahyoid mesenchyme. Frontal sections were screened manually for thyroids and photos were all taken using the same settings (10x objective of the same microscope, same filter set, same field of view, same contrast, and brightness adjustments). Only embryos were analyzed, in which the anlagen (or area with remnant thereof) could be clearly identified. For each embryo and anlage, the section was chosen, which contained the largest cross-sectional area of the thyroid [12]. Relative size of each thyroid was determined by measuring the sectional surface area (in square pixel) using the ImageJ polygon tool [13]. Control and morphant values were graphically represented as a boxplot, and statistical calculations were performed using an unpaired Wilcoxon rank sum test with continuity correction, both using statistical R (www.r-project.org; [14]).

\section{Sanger Sequencing}

All variants in genes present in the cDNA libraries were confirmed by Sanger sequencing as described previously [15]. For sequencing the coding region of TRPC4AP, PCR products of exonic amplicons were generated using Paq5000 Polymerase (Agilent, Santa Clara, CA, USA) and subsequently sequenced (GATC, Konstanz, Germany). All primer sequences used are listed in online supplementary Table S2.

\section{Databases}

TGP (https://browser.1000genomes.org)

EVS (http://evs.gs.washington.edu/EVS/)

gnomAD (https://gnomad.broadinstitute.org)

dbSNP (https://www.ncbi.nlm.nih.gov)

AQUAPE (http://buster.zibmt.uni-ulm.de/hypothyreose)

GTex-Portal (Genotype-Tissue Expression Project; https:// www.gtexportal.org)

PolyPhen 2 (http://genetics.bwh.harvard.edu/pph2/)

MutationTaster (http://www.mutationtaster.org/)

PROVEAN (http://provean.jcvi.org/seq_submit.php)

ANNOVAR (http://annovar.openbioinformatics.org/)

Condel (https://omictools.com/condel-tool)

\section{Results}

\section{Whole Exome Sequencing}

To identify new candidate genes implicated in the etiology of TD, a cohort of 185 patients with $\mathrm{CH}$ due to TD 
Table 3. Summary of candidate genes, analyses in prediction programs und findings in gnomAD browser

\begin{tabular}{|c|c|c|c|c|c|c|c|c|c|c|}
\hline Patient & Gene & Inheritance & $\begin{array}{l}\text { Heterozygosity - } \\
\text { Homozygosity }\end{array}$ & $\begin{array}{l}\text { Position } \\
\text { base exchange }\end{array}$ & $\begin{array}{l}\text { Amino acid } \\
\text { exchange }\end{array}$ & Condel & PPH2 & Provean & SIFT & $\begin{array}{l}\text { Mutation } \\
\text { Taster }\end{array}$ \\
\hline \multirow[t]{4}{*}{1} & CRTC2 & De novo & Heterozygous & chr1:153924583 C>A & p.G303V & $\mathrm{N}$ & PrD & $\mathrm{N}$ & $\mathrm{T}$ & $\mathrm{D}$ \\
\hline & IQCF5 & Inherited & Homozygous & chr3:51907870 C>T & p.R109H & $\mathrm{D}$ & PoD & De & $\mathrm{T}$ & $\mathrm{D}$ \\
\hline & $A N K F N 1$ & Inherited & Homozygous & 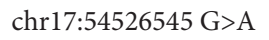 & p.R405Q & $\mathrm{N}$ & PoD & $\mathrm{N}$ & $\mathrm{T}$ & $\mathrm{D}$ \\
\hline & VEZF1 & Inherited & Homozygous & $\operatorname{chr17:56060280~C>T~}$ & p.V170I & $\mathrm{N}$ & B & $\mathrm{N}$ & $\mathrm{T}$ & $\mathrm{D}$ \\
\hline \multirow[t]{2}{*}{2} & SRRM3 & Inherited & Compound heterozygous & chr7:75890695 A>G & p.K201E & - & $\operatorname{PrD}$ & $\mathrm{N}$ & $\mathrm{Da}$ & $\mathrm{D}$ \\
\hline & SRRM3 & Inherited & Compound heterozygous & chr7:75915079 C >A & p.P627H & - & - & $\mathrm{N}$ & $\mathrm{T}$ & $\mathrm{D}$ \\
\hline 4 & FAM127C & Inherited & Hemizygous & chrX:134156480 G>A & p.R4* & - & - & - & - & $\mathrm{D}$ \\
\hline 6 & TRPCAAP & De novo & Heterozygous & chr20:33595385 G>A & p.Q552* & - & - & - & - & $\mathrm{D}$ \\
\hline
\end{tabular}

* Stop codon. Patient 3 revealed no candidate gene and patient 5 demonstrated the disease causing PAX8 mutation and were therefore not included on this table. D, disease causing; Da, damaging; De, deleterious; P, polymorphism; PD, possibly damaging; PrD, probably damaging; $\mathrm{B}$, benign; N, neutral; $\mathrm{T}$, tolerated; -, not interpretable by prediction programs.

was collected. First, WES was performed in 6 probands $(3$ females, 3 males) and their respectives parents (total: 18 samples [6 trios]).

The exonic raw sequence data resulted in 4,994 variants and were mapped to the 1,000 genomes phase II reference genome (hs37d5) followed by a bioinformatical variant detection pipeline (see Material and Methods and Fig. 1). After specific filtering, 650-750 candidate genes (SNV or Indels) were identified per patient.

\section{Mutation Analysis of Known TD Genes}

In the next step, each patient was checked for mutations of known genes associated with TD [4]. In 4 patients, heterozygous variants were found in the FOXE1, $P A X 8, G N A S$, and the TG gene (Table 2). Patient 5 carried 2 heterozygous mutations on the same allele in the PAX8gene transmitted by his mother, who suffered from $\mathrm{CH}$ as well. So these mutations were presumed to be disease causing, and this patient was excluded from further analysis.

\section{Strategies for Identifying Candidate Genes}

About 30-46 variants in 14-30 genes of each patient were identified when the heterozygous variants transmitted by the healthy parents of the 5 remaining patients were eliminated. Seven to 17 genes remained per index patient after excluding the variants with an allele frequency higher than 0.01 in the general population (from gno$\mathrm{mAD}$ ). A rare disease such as $\mathrm{CH}$ is probably not caused by a mutation with a higher allele frequency. These vari- ants were analyzed on their effect on protein function and were considered for further analysis if they were predicted as damaging by at least one of the following prediction tools: SNVs: PROVEAN, MutationTaster, Polyphen 2, SIFT and Condel and indels: PROVEAN, MutationTaster, SIFT, and Indeltype (Fig. 1). The consensus score added up the predictions of the damaging variants in these programs.

In the next step, the selected variants were analyzed regarding their intolerance score [16] and their frequency in the gnomAD browser. A total number of 1-6 genes and an average number of 2.4 candidate genes per patient were identified with a negative intolerance score, with a lower frequency than 5 in the gnom AD browser, consensus score $\geq 1$, and mutation identified as homozygous, heterozygous, compound heterozygous, and de novo (Tables 3,4). The following genes were predicted as potential disease causing genes and selected for Sanger sequencing: TRPC4AP, FAM127C, SCN10A, SRRM3, and CRTC2.

The identified genes were analyzed by the CADD score [17], by the pLI-Score, and by gnomAD browser. After these analyses, the TRPC4AP gene qualified as the most likely disease causing candidate gene (Table 4 ).

Expression of TRPC4AP in the adult human thyroid gland was verified utilizing RT-PCR (Fig. 2a).

\section{Sequencing TRPC4AP in a Large CH Patient Cohort}

The mutation of TRPC4AP found in patient 6 by WES was confirmed by Sanger sequencing to exclude artifacts. 
Table 4. Summary of candidate genes analyzed by CADD score, pLI score, and gnomAD analysis

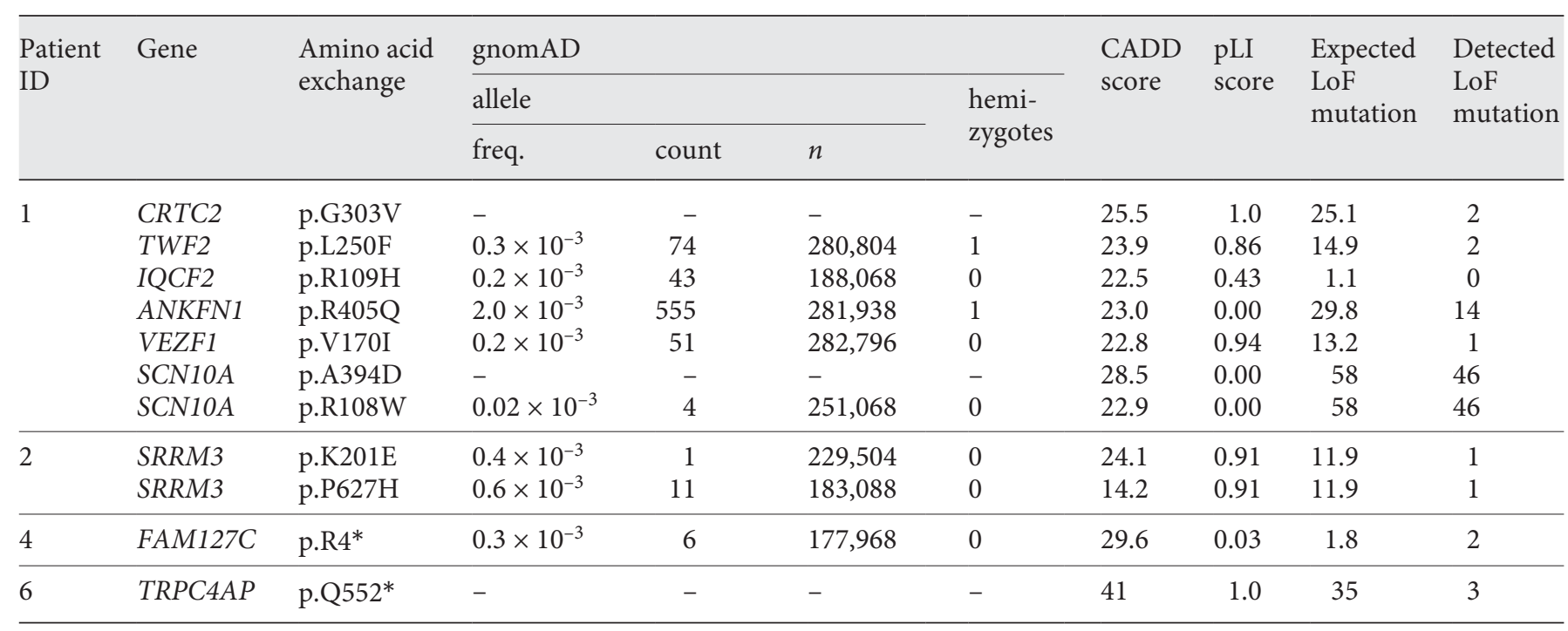

* Stop codon. TRPC4AP, transient receptor potential channel 4-associated protein.

This analysis revealed a de novo mutation in exon 14, which resulted in a stop codon (Table 3). This mutation was neither listed in the dbSNP nor in the gnomAD browser sequence database, demonstrated a consensus score of 6 (predicted as damaging by all used prediction programs) and a negative intolerance score (Tables 3, 4). A potential protein dysfunction may be caused by this mutation.

Thus TRPC4AP was selected for sequence analysis in 179 additional patients with TD to gain further evidence for a causative pathogenic effect in TD. Four further gene mutations in TRPC4AP were identified which might be disease causing according to the predicted damaging potential corresponding to the performed in silico analysis (Table 5).

Two mutations in exon 18 were identified resulting in an amino acids exchange. The first mutation was detected in patient HD-131 c.33591353 resulting in an exchange of the proline at amino acid position 706 to serin (P706S). It was rarely found in the gnomAD browser (allele frequency of 0.0006473 ) and was characterized as disease causing by Mutation Taster and possibly damaging by PolyPhen-2. The second mutation (HD-145) c.33591284 was transmitted by a presumably healthy father and resulted in an exchange of phenylalanin to leucin (F729L). It was found once in the gnomAD browser (allele frequency of 0.0003718 ) and was characterized as disease causing by Mutation Taster and possibly damaging by PolyPhen-2. The mutation in exon 19 (HD-98) c.33591013 resulted in an exchange of the amino acid serine to cysteine (S777C). It was found once in the gnomAD browser in Caucasians, but was characterized as disease causing only by Mutation Taster. The heterozygous mutation in exon $7(\mathrm{Mz}-5)$ resulted in an amino acid exchange of asparagine to serine (N229S). It was found in the gnomAD browser, but was characterized as disease causing by Mutation Taster, but not by PolyPhen-2 (Table 5).

\section{Knockdown of trpc4ap in Xenopus Tadpoles Impairs \\ Normal Thyroid Growth}

To analyze a potential in vivo role of trpc4ap functionally, we turned to the African clawed frog Xenopus laevis. In Xenopus, genes can be manipulated experimentally by microinjection into its large early embryos. We cloned the frog ortholog of trpc4ap and performed an in situ analysis to determine the expression of trpc4ap in the embryonic thyroid anlagen or its precursor tissue. We detected strong signals in the brain and the ventro-lateral area of the pharyngeal foregut in tailbud stages (Fig. 3a, b). At stage 30, expression was also detected in the differentiating pronephros, that is, the amphibian premetamorphic kidney (Fig. 3b). Transversal sections revealed a strong signal in the ventral foregut, where the thyroid anlagen develop from the medial tissue before differentiation and splitting in the 2 lateral anlagen (Fig. 3b; [18]). To perform functional analyses, we designed a $\mathrm{MO}$ oligomer to specifically block translation of trpc4ap (trpc4ap-MO). We injected the trpc4ap-MO into the mesendodermal lineage of 4-cell stage embryos to target the thyroid anlagen. General early development was not strongly impaired, but a subset of morphant tadpoles at stage 45 showed some degree of edema formation exter- 


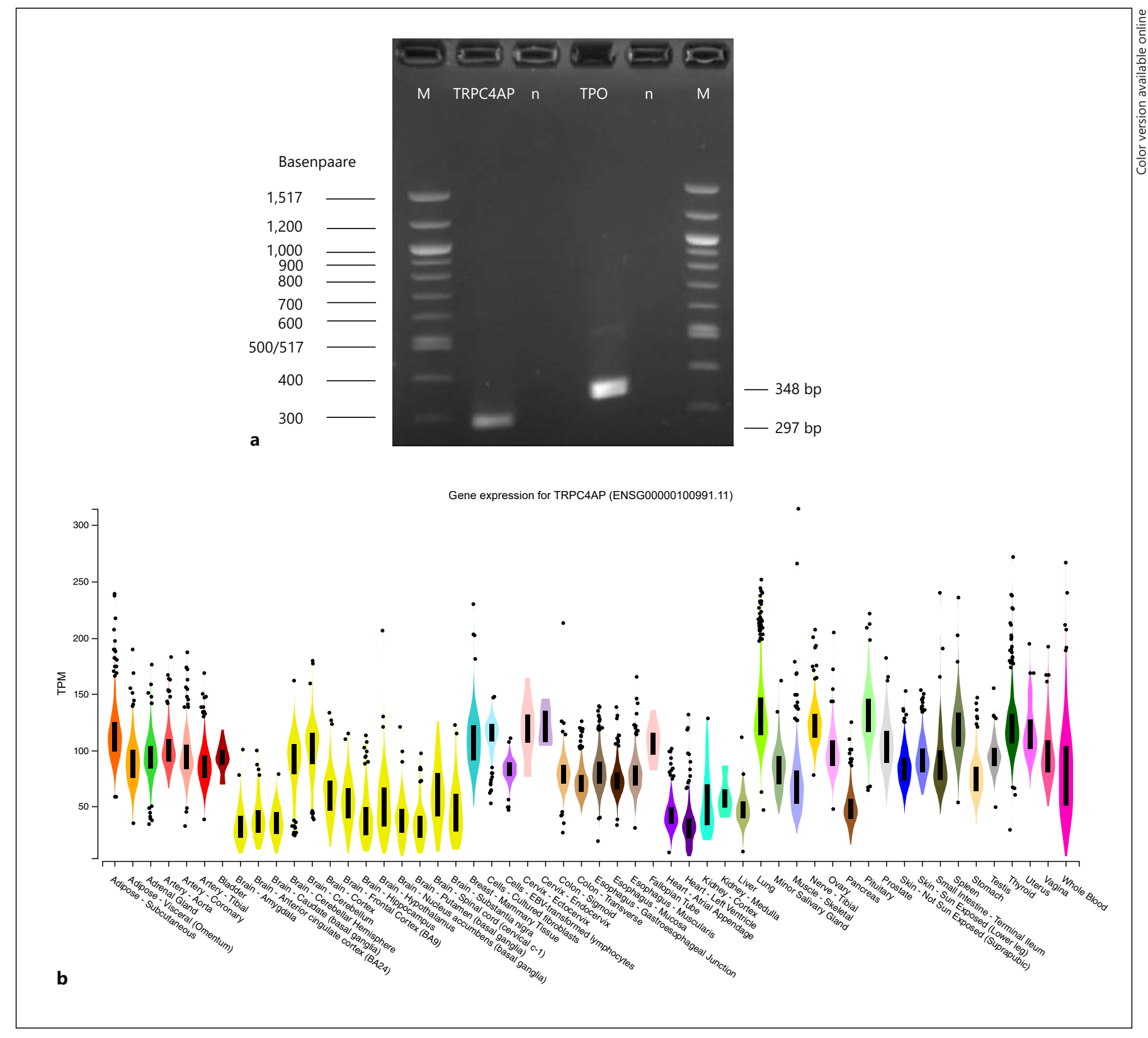

Fig. 2. Expression of TRPC4AP in human thyroid tissues and expression of TRPC4AP using the GTex-Portal. a The bands of TRPC4AP and TPO are depicted. $\mathrm{M}=100 \mathrm{bp}$ marker. $\mathbf{b}$ The Genotype-Tissue Expression Project was supported by the Common Fund of the Office of the Director of the National Institute of Health and by NCI, NHGRI, NHLBI, NIDA, NIMH, and NINDS. Expression of TRPC4AP was not exclusively in the thyroid but highly expressed in the thyroid with 117.2 transcripts per kilobase million. TPO, thyroid peroxidase; TPM, transcripts per kilobase million; TRPC4AP, transient receptor potential channel 4-associated protein.

nally, as compared to same age sibling control specimens (Fig. 3c, e). This was probably caused by partially interfering with normal kidney function, where trpc4ap was active (cf. Fig. 3b). Frontal sections of such embryos were then analyzed for thyroid development. In both, control (Fig. 3d) and morphant (Fig. 3f) embryos, thyroid anlagen was found bilaterally in the medial mesenchyme, but thyroid sizes appeared reduced upon knockdown of trpc4ap. Indeed, quantification revealed an average reduction of thyroid sizes by $21 \%$ in morphant embryos (Fig. $3 g$ ). Next, to verify specific- 
ity of these results, we designed a single-guide RNA (sgRNA) specific for exon 1 of the Xenopus trpc4ap gene to perform "Clustered Regularly Interspaced Short Palindromic Repeats"/Cas9-mediated gene knockout experiments. Embryos were injected at one cell stages with trpc4ap-sgRNA and Cas9 protein, then mutant embryos were again analyzed at stage 45 for effects on thyroid development. Successful genome editing of trpc4ap was verified using T7 Endonuclease-based mutation detection (Fig. 3h). On aver-

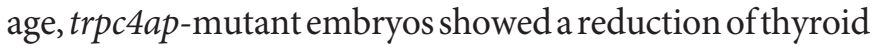
sizes by $41 \%$ (Fig. 3i), as compared to uninjected specimens of the same clutch (Fig. $3 j-m$ ), supporting the effect seen in the knockdown experiments. Also in these analyses, some of the mutant embryos displayed milder signs of edema formation (cf. Fig. 31). These results show that trpc4ap is activated in the thyroid precursor tissue in Xenopus and suggest that its function is required for normal thyroid development in vivo.

\section{Discussion}

In this study, WES was performed in 6 patients with $\mathrm{CH}$ caused by TD and their respective parents, in order to detect variants of known genes and to identify novel candidate genes causing $\mathrm{CH}$. Heterozygous variants were found in the FOXE1, PAX8, GNAS, and the TG gene in 4 patients, one of them carrying $2 P A X 8$ mutations which were transmitted by the affected mother on the same allele. Further potentially damaging variants were detected in 5 others genes not yet related to $\mathrm{CH}$ (TRPC4AP, FAM127C, SCN10A, SRRM3, CRTC2). The most promising variant in TRPC4AP as defined by the applied in silico prediction tools was further analyzed by Sanger sequencing in a large cohort of affected patients with $\mathrm{CH}$ due to TD. In 4 additional patients 4 other mutations were detected in TRPC4AP and were predicted in silico as being damaging. The expression

Table 5. Coding mutations in TRPC4AP from 186 patients

\begin{tabular}{|c|c|c|c|c|c|c|c|c|c|c|c|c|c|c|c|}
\hline \multirow[t]{3}{*}{ Patient } & \multirow[t]{3}{*}{ Exon } & \multirow{3}{*}{$\begin{array}{l}\text { Position } \\
\text { (Chr. 20) }\end{array}$} & \multirow{3}{*}{$\begin{array}{l}\text { Base } \\
\text { exchange }\end{array}$} & \multirow{3}{*}{$\begin{array}{l}\text { Amino acid } \\
\text { exchange }\end{array}$} & \multirow[t]{3}{*}{ RS number } & \multicolumn{4}{|l|}{ GnomAD } & \multirow{3}{*}{$\begin{array}{l}\text { Homozygotes } \\
\text { in cohort }\end{array}$} & \multirow{3}{*}{$\begin{array}{l}\text { Mutation } \\
\text { taster }\end{array}$} & \multirow{3}{*}{ PPH2 } & \multirow[t]{3}{*}{ Provean } & \multirow[t]{3}{*}{ SIFT } & \multirow{3}{*}{$\begin{array}{l}\text { CADD } \\
\text { score }\end{array}$} \\
\hline & & & & & & \multicolumn{3}{|l|}{ allele } & \multirow{2}{*}{$\begin{array}{l}\text { homo- } \\
\text { zygotes }\end{array}$} & & & & & & \\
\hline & & & & & & freq. & count & number & & & & & & & \\
\hline Mz 5 & 7 & 33632487 & $A>G$ & p.N229S & - & $0.04 \times 10^{-3}$ & 12 & 282,770 & 0 & 0 & $\mathrm{D}$ & B & $\mathrm{N}$ & $\mathrm{T}$ & 23.2 \\
\hline HD-6 & 14 & 33595385 & $\mathrm{G}>\mathrm{A}$ & p.Q552* & - & - & - & - & - & 0 & $\mathrm{D}$ & - & - & - & 41.0 \\
\hline HD-131 & 18 & 33591353 & $\mathrm{G}>\mathrm{A}$ & p.P706S & rs200035230 & $0.2 \times 10^{-3}$ & 50 & 279,370 & 0 & 0 & $\mathrm{D}$ & PoD & $\mathrm{N}$ & $\mathrm{T}$ & 22.6 \\
\hline HD-145 & 18 & 33591284 & $A>G$ & p.F729L & rs142961539 & $0.3 \times 10^{-3}$ & 96 & 282,724 & 0 & 0 & $\mathrm{D}$ & PoD & $\mathrm{N}$ & $\mathrm{T}$ & 23.2 \\
\hline HD-98 & 19 & 33591013 & $\mathrm{G}>\mathrm{C}$ & p.S777C & rs117729108 & $2.9 \times 10^{-3}$ & 816 & 281,470 & 13 & 0 & $\mathrm{D}$ & B & $\mathrm{N}$ & $\mathrm{T}$ & 22.1 \\
\hline
\end{tabular}

163 from Heidelberg and 23 from Mainz and the resulting exchange of amino acids are listed; further analyses were carried out by prediction programs. D, disease causing; T, tolerated; N, neutral; B, benign, PoD, possibly damaging; HD, patient from Heidelberg; Mz, patient from Mainz; TRPC4AP, transient receptor potential channel 4-associated protein.

Fig. 3. Knockdown of trpc4ap in Xenopus tadpoles impairs normal thyroid development. a, b In situ hybridization analysis of wildtype trpc4ap expression in tadpole stages of Xenopus laevis. trpc4ap expression in the brain, branchial, and pre-thyroid area (highlighted by black and white arrowheads) of st. 26 (a), and additional expression in the kidney of st. 30 (b) embryos (indicated by black arrowhead). b' Section as indicated by white dashed line in (b) highlights expression of trpc4ap in pre-thyroid and surrounding ventral mesenchymal tissue (black and white arrowheads). c-g In vivo analysis of trpc4ap loss of function showing reduction of thyroid sizes in morphants. c, e Dorsal view of representative phenotypes of whole mount embryos of control (c) or trpc4ap-knockdown embryos (e) at st. 45. $\mathbf{d}, \mathbf{f}$ Representative frontal sections of st. 45 passing through the center of the thyroid anlagen revealing normal sized thyroids in control (d) and reduced sizes in morphant (f) specimen (sections in D and F were stained with "nuclear fast red" for visualization of nuclei; thyroids highlighted by dashed circles; plane of section indicated in c and e). g Quantification of results of MO-injection experiments (as shown in $\mathbf{d}, \mathbf{f}$ ). Data are from 3 independent experiments $(n=3)$. $\mathbf{h}$ T7 Endonuclease I assay of control or trpc4ap-sgRNA/Cas9-injected embryos for verification of trpc4ap genomic editing. T7 was either added (+T7) or left out (-T7) as a technical control. Amplified, noncleaved genomic bands are at 551bp. Expected double bands of 265/286 bp after T7-mediated cleavage were only detected in embryos injected with trpc4ap-sgRNAs and T7 added (right lane, sizes indicated). i Quantification of relative sizes of thyroids from trpc4ap-sgRNA injected or control specimens revealed a very highly significant reduction in average thyroid sizes upon Clustered Regularly Interspaced Short Palindromic Repeats/Cas9-mediated knockout of Xenopus trpc4ap. Data are from 3 independent experiments $(n=$ 3 ). $\mathbf{j}$, I Dorsal view of representative phenotypes of whole mount embryos of control (j) or trpc4ap-knockout embryos (I) at st. 45. $\mathbf{k}, \mathbf{m}$ Representative frontal sections of st. 45 passing through the center of the thyroid anlagen revealing normal sized thyroids in control (k) and reduced sizes in morphant $(\mathbf{m})$ specimen (vibratome sections in $\mathbf{k}$ and $\mathbf{m}$ were taken using dark-field microscopy; thyroids highlighted by dashed circles; plane of section indicated in $\mathbf{j}$ and I). ${ }^{*} p<0.05,{ }^{* *} p<0.01,{ }^{* * *} p<0.001$. bp, base pairs; co, control; $m$, DNA ladder; $n$, number of single thyroid anlagen analyzed for each condition; sgRNA, single-guide RNA; st., stage; T7, T7 Endonuclease I; MO, Morpholino.

(For figure see next page.) 
of TRPC4AP was not restricted to the thyroid (analyzed by GTex-portal https://www.gtexportal.org), but highly expressed in the thyroid with 117.2 transcripts per kilobase million (Fig. 2b), which was validated by RT-PCR in human thyroid tissue originating from a surgically removed thyroid gland of a patient with Graves' disease (Fig. 2a). Specific thyroid expression of TRPC4AP has been previously also detected by exploration of the human thyroid transcriptome [19]. Pathway analyses provided evidence that TRPC4AP is linked to significant transcription factors and peptides which are involved in anlage, proliferation, migration, growth, organization, differentiation, and survival of the thyroid gland and thyroid hormone synthesis (Fig. 4). Therefore, variants in TRPC4AP may be potentially disease causing in $\mathrm{CH}$. TRPC4AP functions as an adapter molecule to IKBKG (NF-kB; see also Fig. 4, Ingenuity Pathway Analysis) and seems to be linked to TSH regulation of thyroid function (TSH receptor, TSHR), to govern key enzymes involved in transmembrane iodine transport (SLC26A4/pendrin, SLC5A5/sodium iodide symporter) and intrathyroidal hormone synthesis ( $\mathrm{Tg}$,

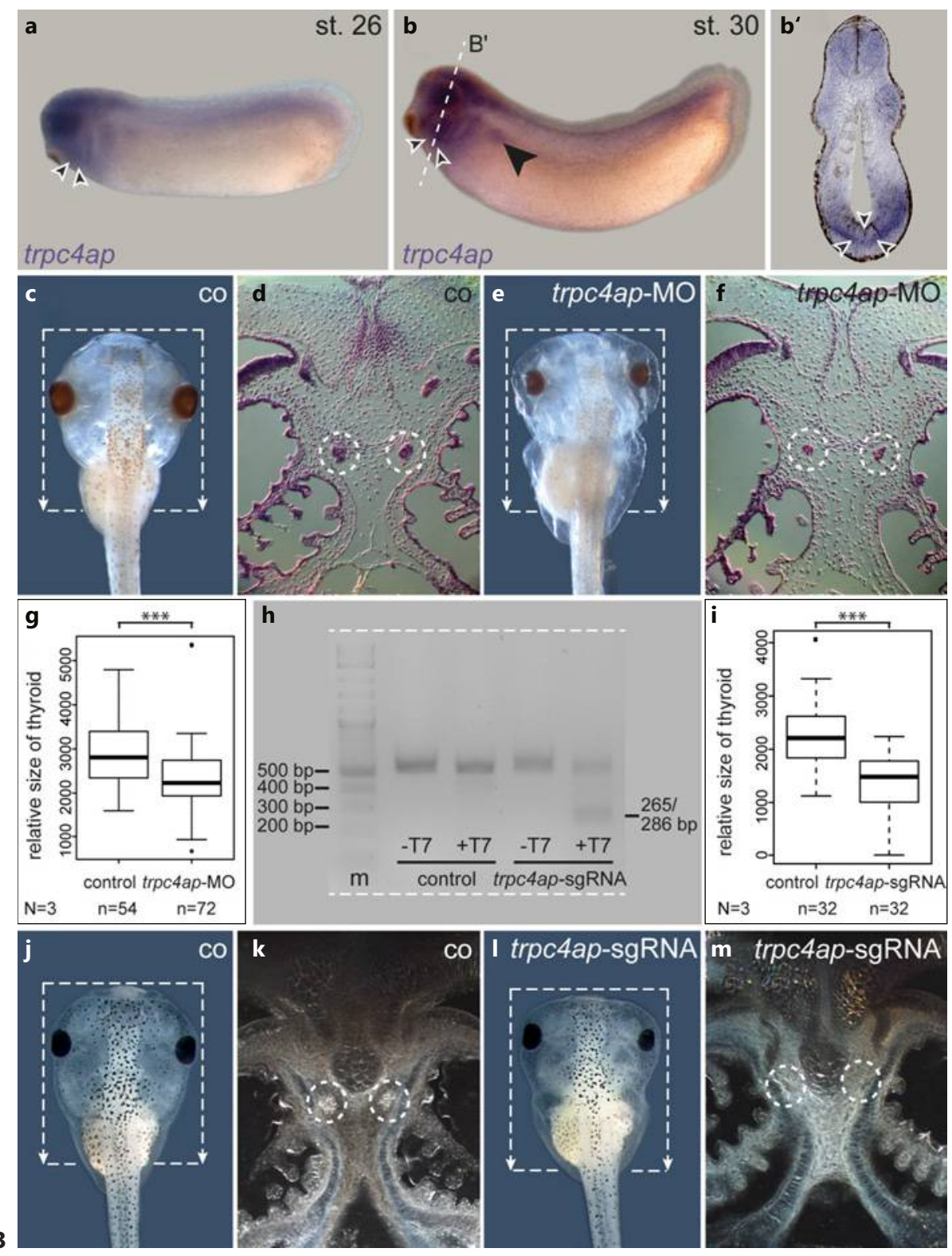




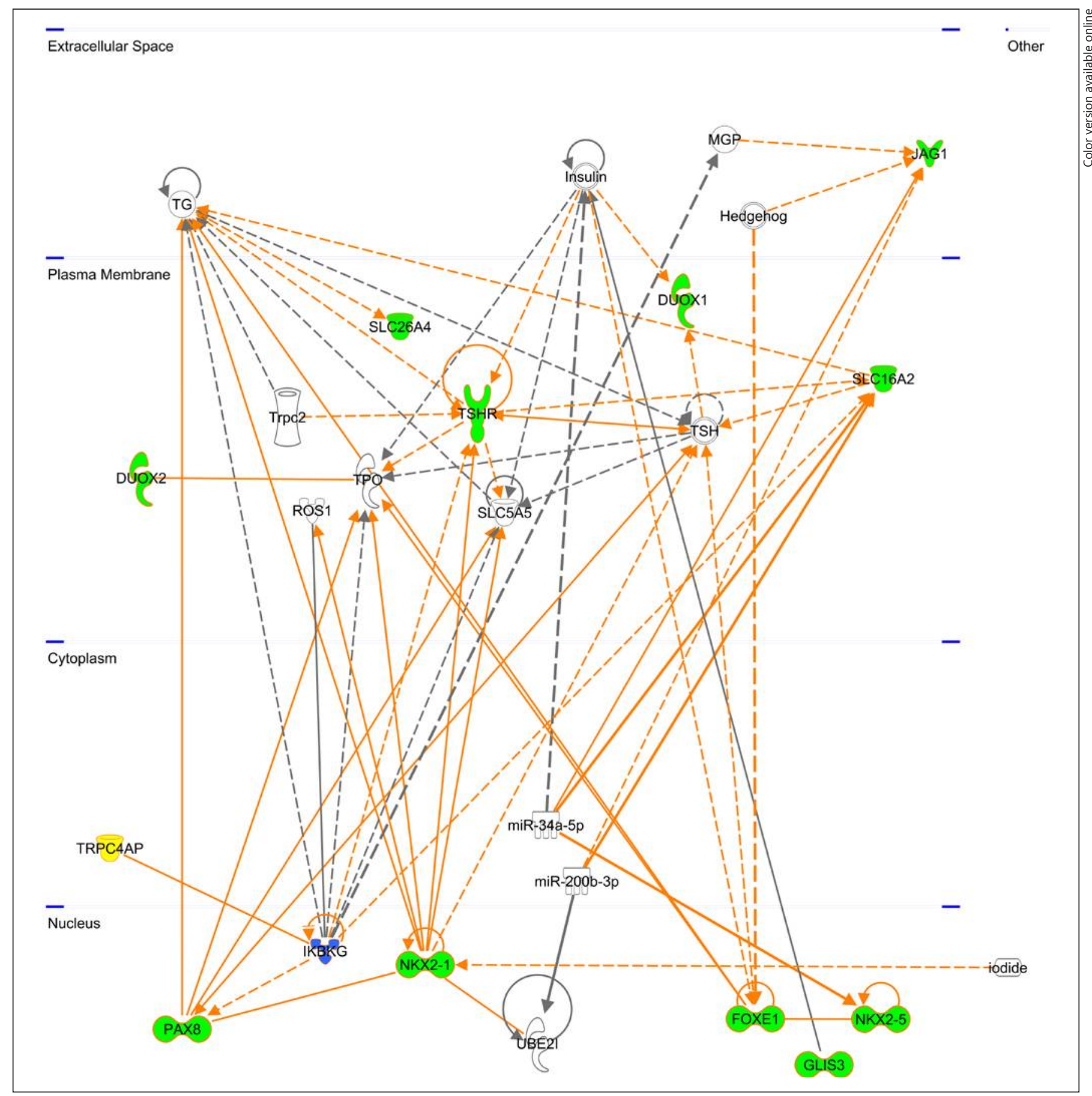

Fig. 4. IPA network analysis. Known genes involved in thyroid gland development (green) and TRPC4AP (yellow) were analyzed using the IPA network analysis. TRPC4AP interacts with IKBKB (blue). TRPC4AP seems to be linked to TSH regulation of thyroid function (TSH receptor), to govern key enzymes involved in transmembrane iodine transport (SLC26A4/pendrin, SLC5A5/sodium iodide symporter) and intrathyroidal hormone synthesis (thyroglobulin, DUOX1/2, thyroid peroxidase) and is related to the transport of thyroxine from blood to cerebrospinal fluid (SLC16A2/MCT-8). Furthermore, TRPC4AP and IKBKG are interconnected directly or indirectly with thyroid transcription factors (NKX2-1/ thyroid transcription factor I, PAX8, FOXE1, GLIS3, and probably NKX2-5) which chaperone the organogenesis of the thyroid gland. TSH, thyroid-stimulating hormone. 
DUOX1/2, TPO) and is related to the transport of thyroxine from blood to cerebrospinal fluid (SLC16A2/ MCT-8). Furthermore, TRPC4AP and IKBKG are interconnected with thyroid transcription factors (NKX2-1 (thyroid transcription factor 1/TTF1), PAX8, FOXE1, GLIS3, and probably NKX2-5) which chaperone the organogenesis of the thyroid gland [20]. Defects in all of these transcription factors and peptides have been detected in patients with $\mathrm{CH}$ due to dysgenesis and dyshormonogenesis [4]. Therefore, variants in regulatory proteins such as IKBKG/TRPC4AP may possibly be involved in the spectrum of developmental anomalies with or without hypothyroidism varying from athyreosis to hypoplasia of an orthotopic gland [2]. In accordance with the high phenotypic variability of TD are our functional analyses in Xenopus laevis using $\mathrm{MO}$ antisense oligomers which revealed reduced sizes of thyroid anlagen by $21 \%$ in trpc4ap morphant and $41 \%$ in mutant embryos. This finding indicates hypoplasia of the thyroid gland as observed in TD with abnormal organogenesis.

The index patient (6) with a heterozygous variant in TRPC4AP presented with unequivocal biochemical characteristics of primary hypothyroidism and ultrasound visualized a thyroid gland in typical location over the thyroid cartilage in the newborn period as measurement of serum thyroglobulin was within reference range (41 ng/ $\mathrm{mL}$ ). During follow-up primary hypothyroidism persisted as the dose of L-thyroxine had to be increased in order to maintain euthyroidism. Thyroidal tissue regression to hyperechogenic remnants in the normal position of the thyroid gland could be visualized by ultrasound at 2 years of age when serum thyroglobulin $(2.8 \mathrm{ng} / \mathrm{mL})$ was low despite elevation of serum TSH (32 IU/L). Thyroidal phenotypes in the neonatal period of the other patients with TRPC4AP variants remain largely obscure as sonographies were not routinely performed right after birth. However, sonographic findings report athyrosis, hypoechogenic, and hyperechogenic thyroidal remnants or a hypoplastic thyroid gland in loco typico as examined during follow-up during childhood. These characteristics match very well to those found in Xenopus laevis using $\mathrm{MO}$ antisense oligomers exhibiting a hypoplastic thyroid anlage (Fig. 3) and to those found in NEMOTS-KO thyrocytes undergoing progressive apoptosis after birth [20]. Moreover, patients with hypohydrotic ectodermal dysplasia develop primary hypothyroidism in infancy [2123].

TRPC4AP is located at $20 \mathrm{q} 11.22$, encoded by 19 exons, and represents an ubiquitous scaffold and signaling pro- tein which is associated with the tumor necrosis factor receptor and mediates activation of the transcription factor NF-kB and interacts as an adapter molecule with components of the I-kB kinase subunit NF-kB essential modulator IKBKG (NEMO) [20, 24-26]. IKBKG functions as a kinase in numerous cell signaling pathways specifically phosphorylating serine or threonine residues that are followed by a proline residue [27].

In mice bearing a thyroid-specific genetic inactivation of IKBKG, development and differentiation of the thyroid gland appear normal during embryonic development but gradually these mice develop hypothyroidism after birth. Histological and molecular analyses indicate that the absence of IKBKG in postnatal thyrocytes results in a dramatic progressive loss of the thyroid gland cellularity, associated with downregulation of thyroid differentiation markers and ongoing apoptosis. In fact, NF-kB controls the expression of thyroid proteins, including sodium iodide symporter, TTF 1/NKX2-1, PAX8, TPO, and TG. It was concluded that IKBKG-dependent signaling is essential for thyrocyte survival and maintenance of thyroid marker expression [20,28]. These findings may also reflect the potential role of the complex TRPC4AP/ IKBKG in the pathophysiology of congenital primary thyroid disease with deteriorating postnatal thyroid function.

The ability to control the proliferative and the antiapoptotic signaling pathways of thyroid neoplastic cells represents the basis of NF-kB function in human differentiated and undifferentiated thyroid cancer by interacting with oncogenic proteins like such as RET/PTC, RAS, and BRAF [29]. More recently, Reale et al. [20] reported the significance of disturbances in the IKBKG complex for thyroid physiology and pathophysiology by means of downregulation of the thyrocyte differentiation and increased apoptosis. Wild type and NEMOTS-KO thyrocytes proliferate following TSH stimulation, whereas NEMOTS-KO thyrocytes also undergo extensive apoptosis. This finding is consistent with previous studies showing that TSH stimulation on thyrocytes triggers both proliferative and apoptotic processes [30].

Previous studies exploring gene variants in $\mathrm{CH}$ failed to identify variants in TRPC4AP in patients with either TD or dyshormogenesis $[4,31]$. However, WES analyses generated a spectrum of mutations in known genes and identified novel genes associated with $\mathrm{CH}$. Inconsistent or negative findings of targeted genetic studies may reflect the complex clinical and molecular heterogeneity of $\mathrm{CH}$ associated with or without syndromic and extrathyroidal manifestations. Expressivity and phenotypic pen- 
etrance may be highly variable. Also the ancestry of the population studied and the applied methods of next generation sequencing may impinge study outcomes [3234]. As molecular mechanisms explaining most cases with TD remain elusive and Mendelian inheritance has been questioned in the etiology of $\mathrm{CH}$, a 2-hit model has been proposed consisting of an inherited genetic component and de novo mutations or somatic mutations or epimutations [35]. In addition, accumulation of rare variants inherited from unaffected parents, which has been reported in patients with congenital heart disease, may also contribute to the molecular pathogenic mechanisms in $\mathrm{CH}$ [36]. Eventually intronic mutations or small partial gene deletions or non-genetic factors have to be considered which escape WES analyses [4].

However, unequivocal study reports point to a significance of TRPC4AP in concert with IKBKG/NEMO in human thyroid physiology and pathophysiology of human thyroid disease. Hypomorphic NEMO mutations have been recognized in children with hypohydrotic ectodermal dysplasia which is clinically characterized by impaired skin development, severe immune, and primary hypothyroidism. These mutations are believed to reduce rather than abolish NF-kB activation [21-23]. These clinical studies report 3 boys and only 1 girl with hypohidrotic ectodermal dysplasia and primary hypothyroidism which was diagnosed between 2 and 4 years of age. At this time, growth consequently slowed down and bone ages were retarded, but psychomotor development was adequate in 3 of the 4 patients. The thyroid gland in one of them was located at the base of the tongue by scintigram, and measurement of serum thyroglobulin value was not reported. Replacement therapy with L-thyroxine in all of them restored euthyroidism and reversed the growth failure. These reports do not mention whether these patients were screened for $\mathrm{CH}$ in the newborn period. Their clinical course with normal psychomotor development at diagnosis of primary hypothyroidism in infancy, however, strongly suggests remnant thyroid tissue and somewhat compensated thyroid function during the first years of life. Overtime mild thyroidal dysfunction probably switched to overt hypothyroidism with apparent clinical symptoms as reported. These clinical courses correspond to a late onset form of $\mathrm{CH}$ with $\mathrm{TD}$ and/or ectopic thyroid gland. The clinical spectrum of primary hypothyroidism comprises mild to severe forms and respective altered thyroid function. Serum measurements range from slight to massive elevations of TSH and normal to low free thyroxine [1]. Reale et al. [20] reported that NEMO signaling is essential for normal postnatal thyroid gland structure and function impacting thyroid proliferation, growth, differentiation, and survival. Thus, experimental and clinical evidence support our findings of TRPC4AP along with IKBKG/NEMO as a novel candidate gene being involved in the etiology of $\mathrm{CH}$.

Overall previous observations together with our findings suggest that differentiation and migration of thyrocytes during embryonic development are preserved in individuals carrying TRPC4AP/IKBKG-NEMO variants, but proliferation and survival of thyrocytes and expression of key enzymes of thyroid hormone synthesis are postnatally progressively damaged. Thus, TRPC4AP not only represents a novel candidate gene in primary hypothyroidism but may also depict a novel concept in its etiology as progressive postnatal thyroid gland apoptosis has not been reported before. However, the significance of TRPC4AP alone and in combination with IKBKG/ NEMO in thyroid function and their contribution to primary hypothyroidism needs to be explored further and should be analyzed in larger cohorts of patients with $\mathrm{CH}$ marked by thyroid tissue or remnants in typical location over the thyroid cartilage. Furthermore, WES, whole genome, or epigenome studies in larger populations and in vitro and in vivo experiments exploring the organogenesis of the thyroid gland are needed to further elucidate the evolution of the thyroid gland and to decipher further candidate genes and their accumulation explaining the basic molecular principles of $\mathrm{CH}$.

\section{Acknowledgment}

The authors thank the patients and their families for their participation in this study. Furthermore, authors would like to thank the colleagues of the Division of Paediatric Endocrinology and Diabetes of the University children's Hospital in Heidelberg for their continuous support.

\section{Statements of Ethics}

Informed consent was given by the parents. All procedures were in accordance with the ethical standards of the responsible committee on human experimentation (institutional and national) and with the Helsinki Declaration of 1975, as revised in 2000. The study was approved by the Ethical Committee of the University Hospital Heidelberg (S-636/2012).

\section{Disclosure Statement}

The authors have no conflicts of interest to declare. 


\section{Funding Sources}

There are no funding sources to declare.

\section{Author Contributions}

D.C., B.E., P.V., G.A.R., J.P., G.F.H., and M.B.: conceived and designed the study. B.E., P.V., P.H., B.W., C.K., and J.K.: performed the experiments. N.P., M.S., K.L., and R.R.: analyzed the data. D.C., M.B., P.V., G.A.R., and P.H.: wrote the article. G.F.H. approved the final version.

\section{References}

1 Bettendorf M. Thyroid disorders in children from birth to adolescence. Eur J Nucl Med Mol Imaging. 2002 Aug;29(Suppl 2):S439-46.

2 Peters C, van Trotsenburg AS, Schoenmakers N. DIAGNOSIS OF ENDOCRINE DISEASE: Congenital hypothyroidism: update and perspectives. Eur J Endocrinol. 2018 Dec; 179(6):R297-317.

3 Fagman H, Nilsson M. Morphogenesis of the thyroid gland. Mol Cell Endocrinol. 2010 Jul; 323(1):35-54.

4 Persani L, Rurale G, de Filippis T, Galazzi E, Muzza M, Fugazzola L. Genetics and management of congenital hypothyroidism. Best Pract Res Clin Endocrinol Metab. 2018 Aug; 32(4):387-96.

5 Carré A, Stoupa A, Kariyawasam D, Gueriouz M, Ramond C, Monus T, et al. Mutations in BOREALIN cause thyroid dysgenesis. Hum Mol Genet. 2017 Feb;26(3):599-610.

6 Stoupa A, Adam F, Kariyawasam D, Strassel C, Gawade S, Szinnai G, et al. TUBB1 mutations cause thyroid dysgenesis associated with abnormal platelet physiology. EMBO Mol Med. 2018 Dec;10(12):e9569.

7 Ellerbroek VL, Bonfig W, Dörr HG, Bettendorf M, Hauffa B, Fricke-Otto S, et al. Longterm Surveillance of Children with Congenital Hypothyroidism: Data from the German Registry for Congenital Hypothyroidism (AQUAPE “Hypo Dok"). Klin Padiatr. 2015 Jul;227(4):199-205.

8 Rimmer A, Phan $\mathrm{H}$, Mathieson I, Iqbal Z, Twigg SR, Wilkie AO, et al.; WGS500 Consortium. Integrating mapping-, assembly- and haplotype-based approaches for calling variants in clinical sequencing applications. Nat Genet. 2014 Aug;46(8):912-8.

9 Wang K, Li M, Hakonarson H. ANNOVAR: functional annotation of genetic variants from high-throughput sequencing data. $\mathrm{Nu}$ cleic Acids Res. 2010 Sep;38(16):e164.

10 Sive HL, Grainger RM, Harland RM. Early Development of Xenopus Laevis. CSHL Press; 2000.

11 Moreno-Mateos MA, Vejnar CE, Beaudoin JD, Fernandez JP, Mis EK, Khokha MK, et al. CRISPRscan: designing highly efficient sgRNAs for CRISPR-Cas9 targeting in vivo. Nat Methods. 2015 Oct;12(10):982-8.

12 Grim KC, Wolfe M, Braunbeck T, Iguchi T, Ohta Y, Tooi O, et al. Thyroid histopathology assessments for the amphibian metamorphosis assay to detect thyroid-active substances. Toxicol Pathol. 2009 Jun;37(4) 415-24.
13 Schneider CA, Rasband WS, Eliceiri KW. NIH Image to ImageJ: 25 years of image analysis. Nat Methods. 2012 Jul;9(7):671-5.

14 Team, R.C. A language and environment for statistical computing. 2013.

15 Simm F, Griesbeck A, Choukair D, Weiß B, Paramasivam N, Klammt J, et al. Identification of SLC20A1 and SLC15A4 among other genes as potential risk factors for combined pituitary hormone deficiency. Genet Med. 2018 Jul;20(7):728-36.

16 Petrovski S, Gussow AB, Wang Q, Halvorsen M, Han Y, Weir WH, et al. The Intolerance of Regulatory Sequence to Genetic Variation Predicts Gene Dosage Sensitivity. PLoS Genet. 2015 Sep;11(9):e1005492.

17 Kircher M, Witten DM, Jain P, O’Roak BJ, Cooper GM, Shendure J. A general framework for estimating the relative pathogenicity of human genetic variants. Nat Genet. 2014 Mar;46(3):310-5.

18 Nieuwkoop PD, Faber J. Normal Table of Xenopus laevis. New York: Garland; 1994.

19 Vitale L, Piovesan A, Antonaros F, Strippoli $\mathrm{P}$, Pelleri MC, Caracausi M. A molecular view of the normal human thyroid structure and function reconstructed from its reference transcriptome map. BMC Genomics. 2017 Sep;18(1):739.

20 Reale C, Iervolino A, Scudiero I, Ferravante A, D'Andrea LE, Mazzone P, et al. NF- $\kappa B$ Essential Modulator (NEMO) Is Critical for Thyroid Function. J Biol Chem. 2016 Mar; 291(11):5765-73.

21 Fryns JP, Chrzanowska K, Van den Berghe H. Hypohidrotic ectodermal dysplasia, primary hypothyroidism, and agenesis of the corpus callosum. J Med Genet. 1989 Aug;26(8):5201.

22 Pike MG, Baraitser M, Dinwiddie R, Atherton DJ. A distinctive type of hypohidrotic ectodermal dysplasia featuring hypothyroidism. J Pediatr. 1986 Jan;108(1):109-11.

23 Pabst HF, Groth O, McCoy EE. Hypohidrotic ectodermal dysplasia with hypothyroidism. J Pediatr. 1981 Feb;98(2):223-7.

24 Soond SM, Terry JL, Colbert JD, Riches DW. TRUSS, a novel tumor necrosis factor receptor 1 scaffolding protein that mediates activation of the transcription factor NF-kappaB. Mol Cell Biol. 2003 Nov;23(22):8334-44.

25 Soond SM, Terry JL, Riches DW. TRUSS, a tumor necrosis factor receptor-1-interacting protein, activates c-Jun $\mathrm{NH}(2)$-terminal kinase and transcription factor AP-1. FEBS Lett. 2006 Aug;580(19):4591-6.
26 Reale C, Zotti T, Scudiero I, Vito P, Stilo R. The NF- $\kappa B$ Family of Transcription Factors and Its Role in Thyroid Physiology. Vitam Horm. 2018;106:195-210.

27 Chiu JC, Ko HW, Edery I. NEMO/NLK phosphorylates PERIOD to initiate a time-delay phosphorylation circuit that sets circadian clock speed. Cell. 2011 Apr;145(3):357-70.

28 Nicola JP, Nazar M, Mascanfroni ID, Pellizas CG, Masini-Repiso AM. NF-kappaB p65 subunit mediates lipopolysaccharide-induced $\mathrm{Na}(+) / \mathrm{I}(-)$ symporter gene expression by involving functional interaction with the paired domain transcription factor Pax8. Mol Endocrinol. 2010 Sep;24(9):1846-62.

29 Pacifico F, Crescenzi E, Mellone S, Iannetti A, Porrino N, Liguoro D, et al. Nuclear factor$\{$ kappa\}B contributes to anaplastic thyroid carcinomas through up-regulation of miR146a. J Clin Endocrinol Metab. 2010 Mar; 95(3):1421-30.

30 De Gregorio G, Coppa A, Cosentino C, Ucci $\mathrm{S}$, Messina S, Nicolussi A, et al. The p85 regulatory subunit of PI3K mediates TSH-cAMPPKA growth and survival signals. Oncogene. 2007 Mar;26(14):2039-47.

31 Szinnai G. Clinical genetics of congenital hypothyroidism. Endocr Dev. 2014;26:60-78.

32 Zou M, Alzahrani AS, Al-Odaib A, Alqahtani MA, Babiker O, Al-Rijjal RA, et al. Molecular Analysis of Congenital Hypothyroidism in Saudi Arabia: SLC26A7 Mutation Is a Novel Defect in Thyroid Dyshormonogenesis. J Clin Endocrinol Metab. 2018 May;103(5):1889-98.

33 Sun F, Zhang JX, Yang CY, Gao GQ, Zhu WB, Han $B$, et al. The genetic characteristics of congenital hypothyroidism in China by comprehensive screening of 21 candidate genes. Eur J Endocrinol. 2018 Jun;178(6):623-33.

34 Fu C, Luo S, Zhang Y, Fan X, D'Gama AM, Zhang X, et al. Chromosomal microarray and whole exome sequencing identify genetic causes of congenital hypothyroidism with extra-thyroidal congenital malformations. Clin Chim Acta. 2019 Feb;489:103-8.

35 Abu-Khudir R, Larrivée-Vanier S, Wasserman JD, Deladoëy J. Disorders of thyroid morphogenesis. Best Pract Res Clin Endocrinol Metab. 2017 Mar;31(2):143-59.

36 Sifrim A, Hitz MP, Wilsdon A, Breckpot J, Turki SH, Thienpont B, et al.; INTERVAL Study; UK10K Consortium; Deciphering Developmental Disorders Study. Distinct genetic architectures for syndromic and nonsyndromic congenital heart defects identified by exome sequencing. Nat Genet. 2016 Sep;48(9):1060-5. 